\title{
Where Do You Want to Meet? Assessment and Evaluation of Virtual Meeting Software
}

\author{
Orvie Dingwall, Mê-Linh Lê, Caroline Monnin, Andrea Szwajcer, and Sherri Vokey
}

\section{Introduction}

Like many other Canadian professional association executives, the Manitoba Association of Health Information Providers (MAHIP) Executive meets on a monthly basis and strives to maximize efficiency and productivity in their meetings. The average Executive meeting length is 60 minutes and the average travel time to and from any inperson meeting doubles the time of the meeting. Securing an efficient alternative to meeting in person therefore became a priority for the MAHIP Executive. Other Canadian Health Libraries Association (CHLA) chapters, and health library executives or committees, have used virtual meeting software when their members were dispersed across entire countries (such as the CHLA/ABSC Board of Directors [1], the Medical Library Association Board of Directors [2], and the Canadian Virtual Health Library Task Force). Literature has shown that virtual meetings have been successful for conference planning [3] in instances where travel costs made national meetings prohibitive [4] and for rural association members [5]. However, it remained uncertain whether the challenges of meeting virtually would outweigh the challenges of travelling across a single city.

The purpose of assessing virtual meeting software was therefore to determine the feasibility, value, and efficiency for MAHIP Executive meetings and to find the easiest, most convenient, and most cost-effective virtual meeting software.

\section{Description}

All current members of the MAHIP Executive work at the University of Manitoba Libraries, where a Skype Premium account is available for use. For this reason, Skype Premium was the first meeting software that was used, and we determined that virtual Executive meetings were a practical option to meeting in person. After several meetings, Skype Premium presented a number of challenges and difficulties and the Executive decided to investigate other virtual meeting software including Google Hangouts, Messages (formerly iChat), VSee, and WebEx. These programs were selected largely based on price (i.e., with a preference for a low cost) and on product reviews that indicated potential for meeting the needs of the Executive. Each member of the Executive used a different platform to attend and evaluate the meetings including: Windows desktop, Mac, iPad, and iPhone. The evaluation criteria were comprised of: (i) ease of sign-up/ registration; ( $i i)$ ease of the administrator to host the meeting and join each participant into the virtual meeting; (iii) ease of participants logging into or joining the virtual meeting; (iv) capacity to share documents or screens; $(v)$ quality of the call, challenges experienced, whether the meeting got "dropped", etc.; and (vi) whether there were any unique or special features. Each meeting software program was evaluated in fall of 2013 and again in February 2014 using the aforementioned criteria.

\section{Outcomes}

Each virtual meeting software was assessed using the six evaluation criteria, and their attributes are compared in Table 1.

\section{Skype}

Skype, released in August 2003, is one of the first free voice-over Internet Protocol (VoIP) software options. To use Skype, users must download the software or application onto their computer or device and create a Skype account with a self-assigned Skype "handle". Users can then search for other contacts by their name or handle. To call or chat with other users on Skype, you must first locate them within Skype and then add them to your contacts via invitation.

To utilize group calling of more than two individuals or locations, a Premium account is required, which was

Orvie Dingwall ${ }^{1}$, Mê-Linh Lê, and Sherri Vokey. Neil John Maclean Health Sciences Library, University of Manitoba, 727 McDermot Ave, Winnipeg, MB, R3E 3P5.

Caroline Monnin. J.W. Crane Memorial Library, University of Manitoba, 2109 Portage Avenue, Winnipeg, MB, R3J 0L3.

Andrea Szwajcer. Carolyn Sifton-Helene Fuld Library, University of Manitoba, St. Boniface Hospital \& Research Campus, 409 Tache Ave, Winnipeg, MB, R2H 2A6.

${ }^{1}$ Corresponding author (e-mail: orvie.dingwall@umanitoba.ca) 


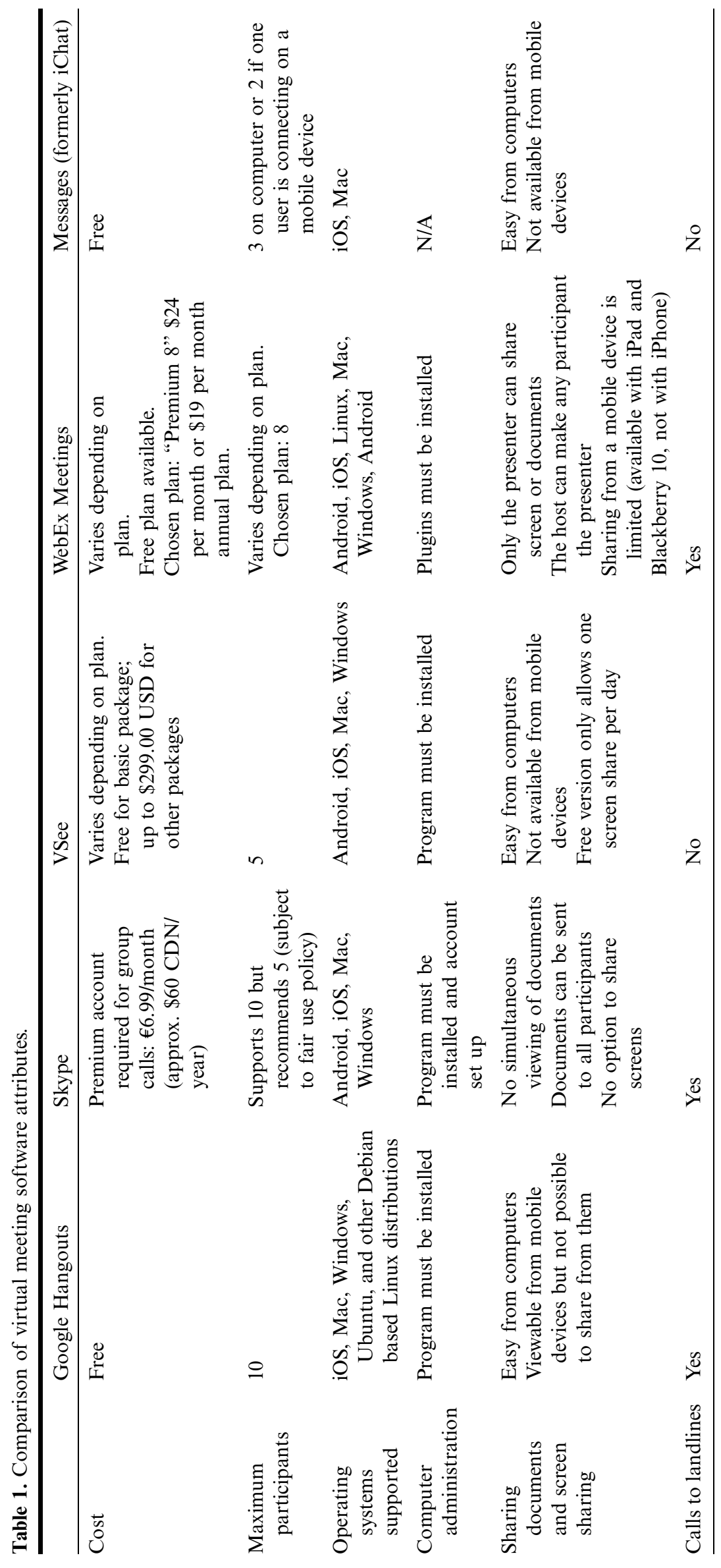


available through the University of Manitoba Health Sciences Libraries. Skype Premium costs $€ 6.99$ per month (approximately $\$ 60.00 \mathrm{CDN}$ per year), and at least one person in the call needs to have a Premium account [6]. Skype Premium facilitates group meetings among three to ten people, though a maximum of five people is recommended for best quality. The interface for group calling is reasonably straightforward. The Premium account holder acts as the host and drags/drops each participant into the call window, and chooses either a video or audio call. Though screen sharing is available using Skype $[7,8]$, we were not able to simultaneously view documents or screens through the software. We were able to send documents to each participant as part of the call, and each participant was required to download and open the document. Skype provides the ability to add contacts by phone number, which is a unique and valuable feature for those not able to download the software.

\section{Google Hangouts}

Google Hangouts, launched in May 2013, is a free video and instant messaging service offered by Google and housed within the Google + social network platform. If a user is already a member of Google + , it is easy to access and start a Hangout. Users who have a Google account (e.g., Gmail) but who are not members of Google + can join by using their Google login. Once signed into Google+, a user's contact list is immediately populated with their Gmail or Google contacts. Users who do not have a Google or Google + account need to register for a free account.

The Hangouts interface is clean and easy to navigate on all platforms. To start a Hangout, users select from the contacts they would like to connect with, and then select whether to connect using instant messaging or video. Up to 10 participants can participate in a Hangout at the same time. Once a Hangout has been initiated, it is not possible to add another contact into the call without ending the active Hangout and starting a new one. It was easy to share documents or screens from a computer (desktop or laptop), but it was not possible to share documents or screens from a mobile device. One of the best features of Hangouts is its ability to recognize who is speaking and make that video window the main focus of the Hangout. There is also the ability to "pin" one user's screen to stay prominent throughout the call (e.g., if they were sharing a document and you wanted to view it through the duration of the discussion).

\section{VSee}

VSee is a low bandwidth telemedicine video conferencing software that provides health access to rural and remote regions. Launched in 2008, VSee utilizes end-toend encryption, that ensures that conversations are private and confidential [9]. This feature facilitated its registration by the United States Food and Drug Administration, and compliance through the Health Insurance Portability and Accountability Act (HIPAA). The basic version of VSee is free to download and use, and there are three additional packages that range from $\$ 9$ to $\$ 299$ USD per user/per month [10]. The paid versions offer access to technical support and increase the amount of screen sharing available. For the purposes of MAHIP Executive meetings, the basic version met the meeting requirements. Signing up for VSee is straightforward and requires an email address. A concern in the sign-up process was VSee's request to access Outlook, Gmail, Hotmail, and Yahoo! to import contacts. Although the request can be bypassed, it felt invasive and was not an issue with the other software.

Creating a meeting and starting a group chat on VSee was simple and intuitive. Users can add colleagues by their email address or import contacts, but recipients must give permission to be added to a contact list. During meetings, participants using a computer can share files or share their desktop, but these features were not available from mobile devices. Throughout the call the screen is shared amongst all participants and every recipient can alter the volume of other participants depending on individual preferences. The picture and sound quality during the Executive meetings were clear, yet users could be easily dropped from a meeting unexpectedly. An added feature of VSee is that users can be added to an ongoing meeting at any time.

\section{Messages (formerly iChat)}

Messages (formerly named iChat) is a Mac OS chat and video-chat program that can host various chat accounts including Google, AIM, Jabber, or an Apple ID. With the release of the Mountain Lion operating system, Apple revamped the iChat program into Messages, which enables users to connect from a Mac to mobile devices with FaceTime and (or) iMessage programs [11]. Users with the older iChat program can connect to those with Messages but not to mobile users. For two individuals to use Messages, they must be using the same host account, for example a user using their Google account cannot connect with a user using an Apple ID.

There are several limitations to Messages that prohibited its use for the virtual executive meetings. Messages can only be used with other Apple products (whether computer, iPhone, or $\mathrm{iPad}$ ), and only when all users are participating through desktop can there be three participants. There is a maximum of two participants when one of the users is connecting with an iPhone or iPad. Consequently, not all executive members would have been able to connect to the meeting. Although sharing documents and sharing a desktop is simple in Messages or iChat, the limitations made it too restrictive for use by the MAHIP Executive.

\section{WebEx}

WebEx is a robust meeting and collaboration tool that allows for high-quality video meetings via desktop computers, mobile devices, and telephones. Features vary according to the four available plans; however, most WebEx Meetings options include VoIP or landline phone audio options; high-definition (HD) video; and desktop, document, and whiteboard sharing. Through the University of Manitoba, the MAHIP Executive had access to the Premium 8 plan, which allows for eight people per meeting and one host. A free WebEx Meetings plan is also available, that can be used with three people and one host, but it would not have been sufficient to connect all the members of the Executive. 
Meetings must be scheduled in advance through the WebEx website, which then automatically generates an email invitation to all participants with a meeting URL and conference number as well as an Outlook Calendar appointment. Audio and HD video quality were excellent across a number of different device types and connections, with no noticeable lags. A chat window is available to all participants, and messages can be sent to the whole group or directed privately to one person. Hosting responsibilities can be transferred among meeting participants, providing anyone the ability to share a desktop, document, or application from their computer and some mobile devices.

Joining the WebEx meeting five minutes before its scheduled start time is recommended to download and install the Cisco WebEx add-ons and complete the speaker and audio tests. Facilitating meetings with WebEx is seamless and efficient once the initial step of browser configuration was completed. It has a professional look and feel and a fully featured interface that includes tools that exceeded the needs of the MAHIP Executive, which offer additional possibilities for collaboration and archiving meeting content.

\section{Discussion}

There are many benefits to holding meetings online, and there are a number of tools available to assist groups to do so efficiently and effectively. The MAHIP Executive felt that although the benefits of meeting online greatly outweighed any disadvantages, effective and efficient meetings are more easily achieved with only some of the platforms that were investigated. Though the five virtual meeting softwares that are outlined in this discussion do not comprise a comprehensive list of the many options available, the selection represents some of the more popular and readily available meeting tools that are currently in widespread use.

There was little success holding meetings with Skype Premium, and after several failed attempts and much frustration it was abandoned. Out of numerous attempts, there was only one instance when the video call was sustained for the duration of the meeting. Skype would often drop the call entirely or default to an audio call with no video, which could have been the result of our university's network and firewalls. Given that there is free software that seems to offer sustained video calls and document sharing, Skype Premium is not recommended.

Messages is also not recommended because of its tight integration with a single operating system (Mac OS/iOS) and inability to host more than three participants.

WebEx was impressive and was undoubtedly the most polished and fully featured of all the virtual meeting tools that were investigated. It allows for the greatest range of access from different devices, landline phones, and operating systems; the highest quality audio and video; and sophisticated whiteboard and screen-sharing options. Though there are several paid plans to choose from, they are not inexpensive. WebEx is therefore recommended with reservations because of the cost, which may be prohibitive for a small, not-for-profit organization.

Google Hangouts and VSee were the clear choices of Executive members due to ease of use, practical functionality, and cost-effectiveness. Though both meeting platforms are free (the version of VSee tested was the free version), they were able to sustain productive and interactive group meetings.

\section{References}

1. Dorgan M. Board meetings via teleconference. CHLA/ABSC $34^{\text {th }}$ Annual General Meeting, Report of the President. 2010 June 10 [cited 2014 March 13]. Available from: http://www. chla-absc.ca/system/files/2010_06_CHLAABSC_AGM_Presi dentReport.pdf.

2. Section Council of the Medical Library Association. Virtual section meetings [Internet]. 2010 June 11 [cited 2014 March 13]. Available from: http://scouncil.mlanet.org/blogs/ news/virtual-section-meetings/.

3. Richards S. Virtual Meetings: Developing a new model for conference committees. College \& Undergraduate Libraries. 2004;11(1):77-82. doi.org/10.1300/J106v11n01_08.

4. Rogers M. ULC holds virtual members' meeting. Library Journal. 2003;128(13):18 [cited 2014 March 13]. Available from: http://lj.libraryjournal.com/2003/08/ljarchives/ulc-holdsvirtual-members-meeting/\#_.

5. Greyson D, Allgaier A. The HLABC WebcastingWebconferencing Pilot Project 3. Journal of the Canadian Health Libraries Association. 2012;33(3):127-30. doi.org/ 10.5596/c12-032.

6. Skype. Premium. [Internet]. Luxembourg: Skype; c2014 [cited 2014 March 9]. Available from: http://www.skype.com/en/ premium/.

7. Skype. How do I share my screen in Skype for Windows desktop? [Internet]. Luxembourg: Skype; c2014 [cited 2014 May 24]. Available from: https://support.skype.com/en/faq/ FA10215/how-do-i-share-my-screen-in-skype-for-windowsdesktop.

8. Skype. How do I share my screen in Skype for Mac OS X? [Internet]. Luxembourg: Skype; c2014 [cited 2014 May 24]. Available from: https://support.skype.com/en/faq/FA10022/ how-do-i-share-my-screen-in-skype-for-mac-os-X.

9. VSee. Simple and Secure Telemedicine [Internet]. Sunnyvale: VSee; c2013 [cited 2014 February 22]. Available from: https:// vsee.com/telemedicine.

10. VSee. Pricing [Internet]. Sunnyvale: VSee; c2013 [cited 2014 February 22]. Available from: https://vsee.com/pricing.

11. Apple. Mac basics: Messages [Internet]. Cupertino: Apple, Inc.; c2014 [modified 21 October 2013, cited 25 February 2014]. Available from: http://support.apple.com/kb/HT5395. 